\title{
Electronic Payment Systems with Fair On-line Verification
}

\author{
FENG BAO, ROBERT DENG, JIANYING ZHOU \\ Kent Ridge Digital Labs, 21 Heng Mui Keng Terrace, Singapore 119613 \\ \{baofeng, deng, jyzhou\}@krdl.org.sg
}

Keywords: e-payment, digital signature, verifiable encryption, fair transaction

\begin{abstract}
Electronic payment systems have been intensively studied in recent years. Generally speaking, e-payment systems can be classified into two categories, i.e., the systems with on-line verification and the systems with off-line verification. The former establishes security under the principle of preventionbefore-the-fact while the latter's security is based on the principle of detectionafter-the-fact. On-line systems are less risky, thus more likely to be adopted by the business community. A critical issue for on-line systems is how to make a transaction fair to each transacting party such that neither party can gain advantages over the other party in a transaction. This can be addressed with a fundamental technique of fair on-line verification. In this paper, we propose a solution to fair on-line verification for electronic payment systems, in which the customer gives verifiable encryption (by a TTP's public key) of the digital money to the shop for on-line verification, instead of giving shop the money itself. The paper not only shows the principle of applying verifiable encryption to e-payment systems, but also focuses on building up a concrete payment system.
\end{abstract}

\section{INTRODUCTION}

\subsection{A Brief Review of Electronic Payment Systems}

Electronic payment systems can be broadly classified into two categories: cashlike systems and bank-card-based systems [AJSW97]. In cash-like or electronic cash

The original version of this chapter was revised: The copyright line was incorrect. This has been corrected. The Erratum to this chapter is available at DOI: 10.1007/978-0-387-35515-3_53 
systems [see for examples, Bra92, Bra93, CST95, CFT98, Cha82, Cha90, CP92, FTY96, FY93, JY96, Oka95, OO91, Sch95], the payer/customer withdraws electronic cash tokens through an account established with a bank before purchases are made.

Bank-card-based electronic payment systems include pay-now systems and paylater systems. In pay-now systems, the payer's account is debited at the time of payment. Examples of such systems are CMU's NetBill [CTS95], USC-ISI's NetCheque [NM95], and FSTC's Electronic Check [FSTC]. In pay-later systems, the payee's bank account is credited the amount of sale before the payer's account is debited. Visa and Master Card's SET [VM97] and IBM's iKP [BGH95] fall into this category.

Of the various requirements for electronic cash systems, one is preserving payer anonymity and the other is preventing double-spending. Maintaining payer anonymity is a double-edged sword. While user anonymity prevents someone from compiling dossiers on individual's spending patterns and whereabouts, it can also be exploited by criminals, e.g., for perfect blackmailing or money laundering. It is this concern which has recently led to the study of anonymity revocation techniques [CMS96, DFTY98, JY96, SPC95, SN92]. A large database of past transactions needs to be maintained in order to detect double-spending.

In bank-card-based or check-like payment systems, a payment is always carried out by sending an information object from the payer to the payee. The object should be self-contained with all information needed to complete a payment. In most electronic cash systems, electronic cash tokens have fixed denomination values and therefore one needs the right combination of tokens to make a payment. This is necessary so that the bank cannot trace the payer by looking at the amount of payment. In check-like systems, the information object issued by the payer carries the exact amount of payment.

\subsection{On-line vs Off-line Verification}

Generally, there are two basic differences between cash-like systems and checklike systems. The first is that in cash-like systems the e-money is the bank's signature while in check-like systems the e-money is the customer's signature. The second difference is that the former facilitates off-line verification while the latter needs on-line verification. For systems with on-line verification, it is prudent to follow the security principle of "prevention-before-the-fact" so that double-spending and over-spending can be prevented and no loss could be made to shops or banks whatsoever. On the other hand, the systems with off-line verification follow the security principle of "detection-after-the-fact". That is, if a customer spends a digital money multiple times, his identity will finally be revealed. However, the loss may have been caused to shops or banks. Therefore, e-payment systems without on-line verification are especially vulnerable against the attacks from malicious enemies who just try to wreck the systems.

Most recently, people began to realize that off-line solution might not be accepted by the financial community [BGJY98, Jak99]. It is worthwhile to further study on-line systems from various angles. This paper considers the fair verification problem for on-line systems. Suppose a customer wants to buy some electronic 
goods from an Internet shop. Obviously it is not a perfect solution if the customer simply gives his e-cash or e-check to the shop for on-line verification before he receives the e-goods. In this paper we propose a method to make the on-line verification fair to both the customer and the shop. Customers need to be protected from malicious shops. Without adequate security mechanisms and measures, it is highly possible for someone to set up a phony store in cyberspace, collect money and disappear without being caught. On the other hand, shops' interests should also be protected. It must be guaranteed that the shops can get the e-money once the online verification is passed and the goods are delivered. To guard the interests of both customers and shops at the same time, what we need is an electronic payment system with the so-called fair transaction property. A system with fair transaction property guarantees that the payer receives the goods if and only if the payee receives valid payment and vice versa.

Our solution proposed in this paper can be applied to both e-cash and e-check systems with on-line verification. Here we demonstrate our solution by constructing an e-check system called DigiCheck. In fact, DigiCheck is more than an electronic payment system. It is a complete electronic transaction system including on-line negotiation, payment and goods delivery. The most outstanding feature of DigiCheck is that it guarantees fair transaction in an efficient and secure manner. This is achieved by the use of a new cryptographic primitive called Verifiable Encryption Scheme (VES).

\section{OVERVIEW OF DIGICHECK SYSTEM}

DigiCheck is designed to allow customers to pay goods and services to shops over the Internet. Parties involved in DigiCheck are 1) TTP; 2) Bank; 3) Customer; 4) Shop.

DigiCheck takes advantage of a recently proposed efficient Verifiable Encryption Scheme (VES) for encryption of digital signatures. A brief introduction to VES is given in the next section. For now, it suffices to say that VES allows anyone to publicly verify that a ciphertext is an encryption of a signature on a given message without disclosing the signature.

After the customer and the shop have successfully negotiated the items to be purchased, the customer signs a check and encrypts the signed check using VES and the TTP's public key. The customer then sends the verifiable encryption of the check, instead of the clear text of the check, to the shop. The shop, using the TTP and the customer's public keys, can verify that the received message is indeed an encryption of the customer's signed check with the right payment amount. The shop then forwards the verifiable encryption to the bank where the received data is similarly verified. If the verification is positive, the bank checks whether the customer's account has sufficient fund to cover the payment. If so, the bank places a hold on the payment amount and replies the shop with a payment approval message. The shop, after receiving the confirmation from the bank, delivers the goods to the customer. Finally, the customer sends the clear text of the check to the shop and the shop forwards it to the bank for crediting his account. 
In DigiCheck, if the customer refuses to send in the clear text of the check after receiving the goods, the shop can bring the verifiable encryption of the check to the off-line TTP. The TTP decrypts the encryption and hands the clear text of the check over to the shop.

\section{VERIFIABLE ENCRYPTION OF DIGITAL SIGNATURE}

Assume that a trustee has a pair of private/public keys for encryption and decryption. Further assume that a prover wants to prove to a verifier the possession of a signature without disclosing it. She can achieve this by the use of VES as follows. The prover encrypts her signature into a ciphertext under the trustee's public key and generates a certificate using VES to convince the verifier that the ciphertext is indeed the encryption of the prover's signature. The verifier has a public verification algorithm to verify the prover's claim based on the ciphertext and the certificate without seeing the signature. If required, the verifier can obtain the prover's signature later by asking the trustee to decrypt the ciphertext.

Generally speaking, a VES of signature is a sort of knowledge proof of owning a signature without having to disclosing it. This topic has been studied recently following two different directions. One approach is the zero-knowledge proof of owning a signature such as in [CD98, FO97, KP98, NBMV99]. The other is the verifiable encryption of signatures as in [ASW98, Ate99, BDM98, BD99, Bao98, BT99, CM98, FO98, Sta96, YY98]. There are basically two differences between the two approaches. First, the former approach uses one-way function as a commitment function while the latter uses a trapdoor one-way function (i.e., a public key encryption scheme) for commitment. As a result, the former emphasizes on demonstrating the ownership of a signature while the latter focuses on the recoverability of the signature. Second, the former approach aims at zero-knowledge proof of owning a signature without disclosing any information about the signature while the latter aims at the computational hardness to get the signature without the decryption key. The latter category can be classified again into two classes. One is for the situation where the receiver himself is the decrypter, like in [BT99, CM98, FO98, Sta96, YY98]. The other is for the situation where the TTP plays the role of decrypter, like in [ASW98, Ate99, BDM98, BD99, Ba098]. For this class the property of against chosen ciphertext attack is very important. The VES we exploit in this paper belongs to the second class.

It is discussed in [ASW98] that there is a VES of signatures for most of the signature schemes. However, for discrete logarithm based signature schemes such as DSA and Schnorr, the VESs presented in [ASW98, Sta96] are not efficient since they use the well-known cut-and-choose technique in the certificate generation. In many electronic commerce applications where a server may deal with large number of on-line transactions, efficiency is a key factor in determining the system's performance and acceptability. The VES presented in [Bao98] is very efficient. The cost of generation and verification is only several exponentiations with exponent size 160-bit. 
Before proceeding to the formal description of VES of signatures, the following notation is listed for easy reference.

$\xi \quad T, P$, and $V$ : the identities of the trustee, prover, and verifier

$\xi$ PKE: a public key encryption scheme

$\xi \quad E_{X}$ and $E_{X}$ : party $X$ 's public and private key pair in a PKE

$\xi \operatorname{encr}\left(m, E_{X}\right)$ : the ciphertext of plaintext message $m$ under the key $E_{X}$

$\xi \operatorname{decr}\left(c, E_{X}\right)$ : the plaintext of ciphertext $c$ under the decryption key $E_{X}$

$\xi \quad$ PKS: a public key signature scheme

$\xi \quad S_{X}$ and $S_{X}$ : party $X$ 's signing and verification key pair in a PKS

$\xi \operatorname{sign}\left(m, S_{X}\right)$ : the signature on message $m$ with signature generation key $S_{X}$

$\xi \quad \operatorname{veri}\left(m, s i g n, S_{X^{-}}\right)$: verification of sign on $m$ with verification key $S_{X-}$

The VES consists of a certificate generation phase by the prover $P$ and a certificate verification phase by the verifier $V$.

\section{Certificate Generation}

The prover $P$ knows a signature on message $m$ under a signature generation key $S_{P}, s:=\operatorname{sign}\left(m, S_{P}\right)$, where $S_{P}$ may be either his own private key or someone else's. $P$ encrypts it under the trustee $T$ 's encryption key $E_{T}$

$$
c:=\operatorname{encr}\left(s, E_{T}\right)
$$

$P$ next generates a certificate, denoted by cert, for proving to $V$ that $c$ is indeed the encryption of $P$ 's signature $s$. Finally, $P$ gives $(c, c e r t, m)$ to $V$.

\section{Certificate Verification}

There is a public verification algorithm cert_veri for $V$ (or anyone else) to check whether $(c$, cert, $m)$ is valid: cert_veri $\left(c\right.$, cert $\left., \bar{m}, E_{T}, S_{P^{-}}\right)=$yes or $n o$. If yes, $(c$, cert, $m)$ is valid; otherwise it is not. If $(c, c e r t, m)$ is valid, $V$ is convinced that $c$ is indeed the ciphertext of $s$. More formally, if

$$
\begin{aligned}
& \text { cert_veri }\left(c, \text { cert, } m, E_{T}, S_{P^{-}}\right)=y e s \\
& \operatorname{veri}\left(m, \operatorname{decr}\left(c, E_{T^{-}}\right), S_{P^{-}}\right)=y e s
\end{aligned}
$$

Hence, if $(c, c e r t, m)$ is valid, $V$ can get signature $s$ (on message $m$ under verification key $\left.S_{P^{-}}\right)$later when necessary by asking $T$ to open $c$.

\section{Security Requirements on VES}

For any message $m$, it is computationally hard to generate $c$ and cert such that (1) holds but (2) does not. It is computationally hard for $V$ to get $s$ from $(c, c e r t, m)$ unless $T$ opens $c$ for $V$. Construction of VES depends on the underlying PKE and PKS being used.

\section{DIGICHECK PROTOCOL}

The DigiCheck protocol aims to provide complete and fair electronic transactions, which covers negotiation of the purchased items, payment, and order fulfillment/delivery. In addition to the notation given in the last section, we use the following notation to represent messages in the protocol.

$\xi \quad C$ : the customer, who has a signing/verifying key pair $S_{C} / S_{C}$ in a PKS

$\xi \quad S$ : the shop, who has a signing/verifying key pair $S_{S} / S_{S^{-}}$in a PKS

$\xi \quad B$ : the bank, who has a signing/verifying key pair $S_{B} / S_{B}$ - in a PKS

$\xi \quad T T P$ : the trusted third party, who has key pair $E_{T T P} / E_{T T P}$ - in a PKE 
$\xi \quad H(X)$ : a one-way hash function applied to message $X$

$T I D$ : a label generated by the shop to identify the transaction

$P R D$ : Product Request Data, used by the customer to specify a product (

$P I D$ : the product description

Product: the digital product, or a non-repudiable invoice

Price: the product price

Salt: a random number generated by the customer

AccountNo_C: customer's bank account number

AccountNo_S: shop's bank account number

$T_{C}$ : the date and time of issuing a check

$T_{B}$ : the expiry date and time of a bank's payment approval

$T_{S}$ : the date and time of check endorsement by the shop

CheckNo: non-decreasing serial number of a check

CheckForm $:=\left(S, H\left(\right.\right.$ Product, Salt), Price, B, AccountNo_C, CheckNo, $\left.T_{C}\right)$ : the content of a digital check to be signed by the customer

$\xi \quad$ Check:= sign $\left(\right.$ CheckForm, $\left.S_{C}\right)$ : a digital check signed by the customer

$\xi$ CipherCheck:= encr(CheckForm, $\left.E_{T T P}\right)$ : a check encrypted with the TTP's public encryption key

$\xi \quad$ Cert: a VES certificate generated by the customer so that cert_veri

(CipherCehck, Cert, CheckForm, $\left.E_{T T P}, S_{C^{-}}\right)=$yes

$\xi \quad$ Approval := sign(CipherCheck, $\left.T_{B}, S_{B}\right)$ : payment approval by the bank

$\xi \quad$ EndorseCheck := $\operatorname{sign}\left(\right.$ Check, $B$, AccountNo_S $\left., T_{S}, S_{S}\right)$ : the shop's endorsement of the check

$\xi \quad$ StopReq $:=\operatorname{sign}\left(\right.$ CipherCheck, $\left.S_{C}\right)$ : customer's request to stop a check

$\xi$ Stop := $\operatorname{sign}\left(\right.$ StopReq, $\left.S_{B}\right)$ : stop of a check confirmed by the bank

We assume that each party either holds the public key certificates of other parties, or is able to retrieve them from a X.509 type of directory service [X509]. We also assume that communication channels between each pair of parties are resilient, i.e., a message inserted into such a channel will eventually be delivered. We further assume that each pair of parties can communicate securely by the use of existing cryptographic protocols (e.g. SSL [SSL]) if they want to maintain the privacy of messages. The DigiCheck protocol is as follows.
1. C to S:
$P R D$, Salt
2. $\mathrm{S}$ to $\mathrm{C}$ :
TID, PID, H(Product, Salt), Price
3. $\mathrm{C}$ to $\mathrm{S}$ :
TID, CheckForm, C, TTP, CipherCheck, Cert
4. $S$ to $B$ :
TID, CheckForm, C, TTP, CipherCheck, Cert
5. B to $\mathrm{S}$ :
TID, $T_{B}$, Approval
6. $\mathrm{S}$ to $\mathrm{C}$ :
TID, Product
7. $\mathrm{C}$ to $\mathrm{S}$ :
TID, Check
8. $\mathrm{S}$ to $\mathrm{B}$ :
TID, Check, B, AccountNo_S, $T_{S}$, EndorseCheck

If the customer did not receive the product in time after sending out the encrypted check CipherCheck at Step 3, the customer can initiate the following check-stopping sub-protocol.

3.1. C to B: TID, CheckForm, C, TTP, CipherCheck, Cert, StopReq

3.2. B to C: $\quad T I D$, Stop 
If the shop did not receive the check from the customer after sending out the product at Step 6, the shop will initiate the following recovery sub-protocol before proceeding to Step 8 .

\subsection{S to TTP: TID, CheckForm, CipherCheck, Product, Salt}

7.2. TTP to S: TID, Check

\subsection{TTP to C: TID, Product}

Steps 1 and 2 represent the negotiation phase of a transaction, in which the customer requests the quotation of a product from the shop. After receiving a product request $(P R D)$ from the customer, the shop will assign a label $(T I D)$ to the on-going transaction, and provide the customer with the product description (PID) and price. These two steps may be repeated as needed until the customer and the shop agree on a price. If two parties cannot reach an agreement, either party can abort the transaction without any disputes.

Once the customer and the shop have negotiated a price for the product, the customer can direct the shop to deliver the product by supplying an encrypted check CipherCheck and a certificate which makes CipherCheck publicly verifiable. The content of the check includes the payee's name, the amount to be paid, the payer's account number, the bank name, the check number and the issue date and time of the check. The digest of the salted product H(Product, Salt) is also included for two reasons.

$\xi \quad$ The shop can verify whether the check is for the payment of the product.

$\xi$ The bank will not know the product that the customer paid for.

The shop will verify the encrypted check based on VES to see if the payee's name and the amount of payment as well as the digest of the slated product are correct. If not, the shop may send an error message to the customer, and the transaction terminates without disputes.

To prevent the customer's overdraft, the shop forwards the message received in Step 3 to the bank for approval. The bank will make the following verification upon the shop's request at Step 4: it checks the check number and rejects a stopped check or the replay of an old check; it checks the issue date of the check and rejects a stale check; it checks the balance of the customer's account and rejects an overdraft check.

Only if all of the above checks are successful, will the bank authorize the transaction and hold on the amount of payment until the unencrypted check is received or the payment approval expires.

After receiving the payment approval from the bank, the shop will deliver the product to the customer at Step 6.

In the normal case, the customer will send the cleartext check to the shop after receiving the product. The shop then endorses the check, in which the shop's bank account number is specified for crediting, and sends the endorsed check to the bank for crediting its account.

If the shop did not receive the cleartext check in time, the shop may bring the encrypted check and the digital product (or a non-repudiable invoice for the physical product) to the off-line TTP. The TTP will 1) decrypt the encrypted check; 2) verify whether the check is for the payment of the product; 3 ) send the cleartext check and the product to the shop and the customer respectively if the above verification is positive. 
Then the shop can continue Step 8 of the protocol and deposit the endorsed check into its bank account.

As the bank will unfreeze the amount of fund when the payment approval expires, if the shop cannot deposit the endorsed check to the bank in time, there may be no sufficient fund in the customer's account to cover the payment. The shop should be aware of the risk, and make sure that there is sufficient time to obtain the cleartext check (either from the customer or from the TTP) before sending the product to the customer at Step 6.

As for the customer, the time of receiving the product will be partly out of his/her control after sending the encrypted check to the shop at Step 3. The shop may postpone sending the encrypted check to the bank for approval (probably because the product is temporarily out of stock) as long as the encrypted check is not regarded as stale by the bank. The check stopping sub-protocol can partly solve this problem. If the shop delays seeking approval of the encrypted check, the customer may stop the check at the bank, thus aborting the transaction safely.

It is important to note that the shop must first obtain the payment approval from the bank before sending out the product at Step 6 or initiating the recovery subprotocol. Otherwise, the bank may not be able to credit the shop's account when the shop deposits the check for clearance, probably because the check has been stopped by the customer, or there is no sufficient fund in the customer's account.

\section{E-CASH WITH ON-LINE VERIFICATION}

In the above we presented an e-check system. Here we briefly explain that the same method can also be applied to cash-like systems with on-line verification. As we mentioned earlier, banking community has shown more interests in on-line systems. Some cash-like systems with on-line verification are being studied [Jak99].

The principle of applying VES to cash-like systems is similar to what we did for DigiCheck. The only difference is that this time the VES is applied to the bank's signature, i.e., to the digital cash that the customer withdraws from the bank. One thing worth mentioning is that the VES used here must be "labeled VES", as studied in [BD99]. Otherwise it is not secure.

\section{REFERENCES}

[AJSW97] N. Asokan, P. A. Janson, M. Steiner and M. Waidner, "The state of the art in electronic payment systems", IEEE Computer, pp. 28-35, September 1997.

[ASW98] N. Asokan, V. Shoup and M. Waidner, "Optimistic fair exchange of digital signatures", Proceedings of Eurocrypt'98, LNCS, Springer-Verlag, 1998.

[Ate99] G. Ateniese, "Efficient verifiable encryption (and fair exchange) of digital signatures", Proceedings of the 6th ACM Conference on Computer and Communications Security, pp. 138-146, 1999.

[BDM98] F. Bao, R. H. Deng and W. Mao, "Efficient and practical fair exchange protocols with off-line TTP", Proceedings of the 1998 IEEE Symposium on Security and Privacy, IEEE Computer Press, Oakland, CA, pp. 77-85, 1998. 
[BD99] F. Bao and R. H. Deng, "Efficient and practical fair exchange protocols with semi-trusted off-line third party", Proceedings of the International Workshop on Cryptographic Techniques \& E-Commerce, Hong Kong, pp. 37-47, 1999.

[Ba098] F. Bao, "An efficient verifiable encryption scheme for the encryption of discrete logarithms", Proceedings of CARDIS'98, LNCS, Springer-Verlag, 1998.

[BGH95] M. Bellare, J. A. Garay, R. Hauser, A. Herzberg, H. Krawczyk, M. Steiner, G. Tsudik and M. Waidner, "iKP - a family of secure electronic payment protocols", http://www.zurich.ibm.com/Technology/ Security/extern/ecommerce.

[BGJY98] M. Bellare, J. Garay, C. Jutla and M. Yung, "VarietyCash: a multi-purpose electronic payment system", Proceedings of the 3rd Usenix Workshop on Electronic Commerce, 1998.

[BT99] F. Boudot and J. Traore, "Efficient publicly verifiable secret sharing schemes with fast or delayed recovery", Proceedings of ICICS'99, LNCS, 1999.

[Bra92] S. Brands, "Untraceable off-line cash based on the representation problem", Technical Report CS-R9323, Centrum voor Wiskunde en Informatica, 1993.

[Bra93] S. Brands, "An efficient off-line electronic cash system based on the representation problem", Technical Report of CWI, CS-R9323, pp. 1-77, 1993.

[CD98] R. Cramer and I. Damgard, "Zero-knowledge proofs for finite field arithmetic or: can zero-knowledge be for free?", Proceedings of Crypto'98, LNCS, 1998.

[CMS96] J. Camenisch, U. Maurer and M. Stadler, "Digital payment systems with passive anonymity-revoking trustees", Proceedings of ESORICS'96, LNCS 1146, Springer-Verlag, pp. 33-43, 1996.

[CM98] J. Camenisch and M. Michels, "A group signature scheme with improved efficiency", Proceedings of Asiacrypt'98, LNCS 1514, pp. 160-174, 1998.

[CST95] L. Camp, M. Sirbu and D. Tygar, "Token and notational money in electronic commerce", Proc of 1st Usenix Workshop on Electronic Commerce, pp. 1-12.

[CFT98] A. Chan, Y. Frankel and T. Tsiounis, "Easy come-easy go divisible cash", Proceedings of Eurocrypt'98, LNCS, Springer-Verlag, pp. 561-575, 1998.

[Cha82] D. Chaum, "Blind signature for untraceable payments", Proceedings of Crypto'82, Plenum Press, pp. 199-203, 1983.

[Cha90] D. Chaum, "On-line cash checks", Proceedings of Eurocrypt'89, LNCS 434, Springer-Verlag, pp. 288-293, 1990.

[CP92] D. Chaum and T. P. Pedersen, "Wallet databases with observers", Proceedings of Crypto'92, LNCS 740, Springer-Verlag, pp. 89-105, 1993.

[CTS95] B. Cox, D. Tygar, and M. Sirbu, "NetBill security and transaction protocol", Proc of the 1st Usenix Workshop on Electronic Commerce, pp. 77-88, 1995.

[DFTY98] G. Davida, Y. Frankel, Y. Tsiounis and M. Yung, "Anonymity control in e-cash systems", Proceedings of Financial Cryptography'98, LNCS, 1998.

[FTY96] Y. Frankel, Y. Tsiounis and M. Yung, "Indirect discourse proofs: achieving efficient fair off-line e-cash", Proceedings of Asiacrypt'96, LNCS 1163, Springer-Verlag, pp. 286--300, 1996.

[FY93] Y. Franklin and M. Yung, "Secure and efficient off-line digital money", Proceedings of ICALP'93, LNCS 700, Springer-Verlag, pp. 165-276, 1993.

[FO97] E. Fujisaki and T. Okamoto, "Statistical zero knowledge protocols to prove modular polynomial relations", Proceedings of Crypto'97, LNCS 1294, Springer-Verlag, pp. 16-30, 1997.

[FO98] E. Fujisaki and T. Okamoto, "A practical and provable secure scheme for publicly verifiable secret sharing and its application", Proceedings of Eurocrypt'98, LNCS, Springer-Verlag, pp. 32-46, 1998.

[FSTC] http://www.fstc.org

[JY96] M. Jakobsson and M. Yung, "Revokable and versatile e-money", Proceedings of the 3rd ACM Symposium on Computer and Communication Security, 1996. 
[Jak99] M. Jakobsson, "Mini-cash: a minimalistic approach to e-commerce", Proceedings of $P K C^{\prime}$ 99, LNCS 1560, Springer-Verlag, pp. 122-135, 1999.

[KP98] J. Kilian and E. Petrank, "Identity escrow", Proceedings of Crypto'98, LNCS, Springer-Verlag, 1998.

[NM95] B. Neuman and G. Medvinsky, "Requirements for network payment: the NetCheck perspective", Proceedings of IEEE Compcon'95, San Francisco, 1995.

[NBMV99] Q. K. Nguyen, F. Bao, Y. Mu and V. Varadharajan, "Zero-knowledge proofs of possession of ElGamal-like digital signatures and it applications", Proceedings of ICICS'99, LNCS, Springer-Verlag, 1999.

[Oka95] T. Okamoto, "An efficient divisible electronic cash scheme", Proceedings of Crypto'95, LNCS 963, pp. 438-451, 1995.

[0091] T. Okamoto and K. Ohta, "Universal electronic cash", Proceedings of Crypto'91, LNCS, pp. 324-337, Springer-Verlag, 1992.

[Sch95] L. Schoemaker, "An efficient electronic payment system withstanding parallel attacks", Technical Report of CWI, CS-R9522, 1995.

[Sta96] M. Stadler, "Publicly verifiable secret sharing", Proceedings of Eurocrypt'96, LNCS 1070, Springer-Verlag, pp. 190-199, 1996.

[SPC95] M. Stadler, J. M. Piveteau and J. Camenisch, "Fair blind signatures", Proceedings of Eurocrypt'95, LNCS, Springer-Verlag, pp. 209-219, 1995.

[SN92] B. von Solms and D. Naccache, "On blind signatures and perfect crimes", Computers and Security, 11(6):581-583, 1992.

[SSL] K. Hichman, "The SSL protocol", Internet Draft <draft-hickman-netscape-ssl00.txt>, April 1995.

[Tyg96] J. D. Tygar, "Atomicity in electronic commerce", Proceedings of the 15th ACM Symposium on Principles of Distributed Computing (PODC'96), pp. 8-26, 1996.

[VM97] Visa and Master Card, "SET secure electronic transaction specification.

[X509] CCITT, "Recommendation X.509: The directory - Authentication framework".

[YY98] A. Young and M. Yung, "Auto-recoverable auto-certifiable cryptosystems", Proceedings of Eurocrypt'98, LNCS, Springer-Verlag, pp. 17-31, 1998. 